Fetal Diagnosis

and Therapy

\title{
Evaluation of Fetal Vocal Cords to Select Candidates for Successful Fetoscopic Treatment of Congenital High Airway Obstruction Syndrome: Preliminary Case Series
}

\author{
J.M. Martínez ${ }^{a} \quad$ M. Castañón ${ }^{b} \quad$ O. Gómez ${ }^{a} \quad$ J.Prat $^{b} \quad$ E. Eixarch ${ }^{a} \quad$ M. Bennasar ${ }^{a}$ \\ B. Puerto ${ }^{a} \quad$ E. Gratacós ${ }^{a}$ \\ a Maternal-Fetal Medicine Department, Institut Clínic de Ginecologia, Obstetrícia i Neonatologia, Hospital Clínic, \\ Institut d'Investigacions Biomèdiques Augusto Pi i Sunyer, University of Barcelona, and Centro de Investigación \\ Biomédica en Red de Enfermedades Raras, and ${ }^{b}$ Hospital Sant Joan de Déu, Barcelona, Spain
}

\section{Key Words}

Congenital high airway obstruction syndrome $\cdot$ Laryngeal atresia $\cdot$ Fetal vocal cords $\cdot$ Fetal echogenic lungs $\cdot$ Hydrops fetalis · Fetal therapy · Fetal surgery

\begin{abstract}
Objective: Congenital high airway obstruction syndrome (CHAOS) normally has a dire prognosis due to laryngeal atresia and association with other anomalies. However, rare cases with an isolated tracheal membrane have been described. We assessed systematically the larynx and vocal cords in fetuses with CHAOS, with the goal of identifying such cases and attempting fetoscopic deobstruction. Methods: Between 2008 and 2012, 7 cases of CHAOS were referred to our institution. The ultrasonographic aspect of the larynx and trachea was assessed. We report the ultrasound findings, necropsy findings of 6 cases and perinatal outcome in 1 case undergoing therapy. Results: In 6 cases, laryngeal atresia was presumed by ultrasound and confirmed by necropsy after termination of pregnancy. In 1 case, normal appearance of the vocal cords led to suspicion of a possible isolated tra-
\end{abstract}

cheal web completely occluding the trachea. Fetoscopy confirmed the finding, thus the membrane was perforated. Lung size normalized and hydrops resolved. A normal fetus was delivered at term, requiring no respiratory support. At 9 months of age, the infant showed a normal laryngoscopy and was developing well. Conclusions: Systematic ultrasound assessment of the vocal cords can be performed in fetuses with CHAOS. We report the first case of long-term intact survival after fetoscopic airway deobstruction.

Copyright $\odot 2013$ S. Karger AG, Basel

\section{Introduction}

Congenital high airway obstruction syndrome (CHAOS) is an extremely rare congenital anomaly mainly caused by laryngeal or tracheal atresia [1]. Obstruction of airways leads to entrapment of fluid within the tracheobronchial tree with secondary pulmonary hyperplasia $[1,2]$. Prenatal ultrasound findings include severely bilateral enlarged echogenic lungs, dilated and fluid-filled trachea and bronchi, and flattening or inversion of the

\section{KARGER}

E-Mail karger@karger.com

www.karger.com/fdt
(C) 2013 S. Karger AG, Basel

$1015-3837 / 13 / 0342-0077 \$ 38.00 / 0$
Eduard Gratacós

Department and Research Center of Maternal-Fetal Medicine

Hospital Clínic-Maternitat

Sabino de Arana 1, ES-08028 Barcelona (Spain)

E-Mail gratacos@ clinic.ub.es 
diaphragmatic convexities. The heart appears small and squeezed in the middle of the thorax due to compression by the lungs. Elevated intrathoracic pressure causes decreased venous return and fetal cardiac failure leading to ascites, placentomegaly, and eventually hydrops fetalis. Perinatal death is the most common outcome $[1,2]$. Though the risk of chromosomal anomalies is extremely low, the association with nonchromosomal syndromes is high [1-3].

If a confident diagnosis is made prenatally, an ex utero intrapartum treatment (EXIT) procedure at the time of planned cesarean section may help establish a functional airway [1]. Despite such therapeutic attempts, CHAOS often results in neonatal demise shortly after birth, as shown in a recent review by Cavoretto et al. [2]. In a recent report, Roybal et al. [4] aimed to identify prenatal characteristics predictive of a milder postnatal course. The absence of hydrops was associated with less adverse outcome, although in general all survivors suffered severe morbidity, with breathing, feeding and speech limitations [4]. Given the poor reported outcome, several attempts to permeate the airways by means of open surgery or fetoscopy have been reported [5-8]. Initial attempts [5-7] were performed in cases with complex forms of laryngeal atresia and either died during the procedure or suffered severe sequelae. In the most recently reported case, Kohl et al. [8] reported a purely fetoscopic approach to achieve tracheal decompression in a fetus with apparently isolated tracheal obstruction, although the infant eventually died.

Although laryngeal atresia seems to be the rule in CHAOS, airway obstruction may rarely be the consequence of an isolated stenosis, or even a membrane-like web [9]. In these fetuses, laryngeal anatomy is preserved and consequently deobstruction should lead to full recovery of normal lung development. A potential difficulty may be the prenatal identification of such cases. It has been claimed that these variations could be undistinguishable on ultrasound $[2,10,11]$. In general, however, no attempts were made to visualize the ultrasound appearance of the vocal cords $[2,4,10,11]$. As part of our clinical experience in the fetoscopic therapy [i.e. fetal endoscopic tracheal occlusion (FETO)] for congenital diaphragmatic hernia $(\mathrm{CDH})$ [12], we acquired the ability to visualize with high accuracy the fetal trachea and vocal cords. We postulated that systematic visualization of the laryngeal area in CHAOS would allow to visualize variations in the anatomy, and consequently to pick up cases that might eventually benefit from a fetoscopic intervention.
In this study, we describe our initial experience with such an approach in 7 cases and report the successful use of fetoscopy in 1 case with an isolated membrane as the cause of CHAOS.

\section{Materials and Methods}

We report a clinical series prospectively collected among fetuses referred to our Fetal Medicine Unit with a diagnosis of CHAOS, from September 2008 to September 2012. Gestational age at examination was determined by first-trimester fetal crown-rump length measurement. The study protocol was approved by the local ethics committee and all patients provided informed consent for the use of the images for clinical studies.

Ultrasound examinations were performed using high-resolution equipment, either a Siemens Sonoline Antares (Siemens Medical Systems, Malvern, Pa., USA) or a Voluson 730 Expert/E8 (GE Healthcare Technologies, Milwaukee, Wisc., USA) ultrasound machine, with 6-2- and 7-4-MHz curved-array probes.

A thorough evaluation by experienced specialists in fetal medicine including ultrasound, echocardiography and, in some cases, magnetic resonance imaging was performed. The diagnosis of CHAOS was based on typical findings in all cases: massive bilateral enlargement with increased echogenicity of the lungs, compression of the fetal heart and flattened or everted diaphragms, with dilation of the trachea and main bronchi. The presence of isolated ascites or hydrops fetalis was noted as well.

Thereafter, particular attention was paid to the vocal cords. For these purposes, a coronal view at the level of the fetal neck, allowing to visualize the superior aspect of the trachea, the larynx and vocal cords, and the lower aspect of the pharynx. We systematically assessed the presence or absence of anatomical and functional normal features, including a normal appearance, and movements of opening-closure, with passage of fluid through the trachea by color Doppler (fig. 1). All cases were assessed by two or more experienced examiners. Confirmation about the diagnosis of CHAOS and the characteristics of the larynx was obtained by necropsy in most cases, following termination of pregnancy. In the surviving fetus, postnatal evaluation with laryngoscopy was performed.

\section{Results}

During the 5-year study period, 5 pregnant women diagnosed with CHAOS were referred. Median maternal age and gestational age on referral were 33 years (range 22-40) and 20 weeks (range 15-26). At presentation, 6 fetuses had signs of hydrops fetalis and 1 had only ascites. Three fetuses were female and 4 male. The larynx could be reliably visualized in all cases.

In the first 6 cases, a characteristic appearance of the larynx was observed, suggesting the presence of laryngeal atresia/dysplasia (fig. 2). With the upper end of the tra- 

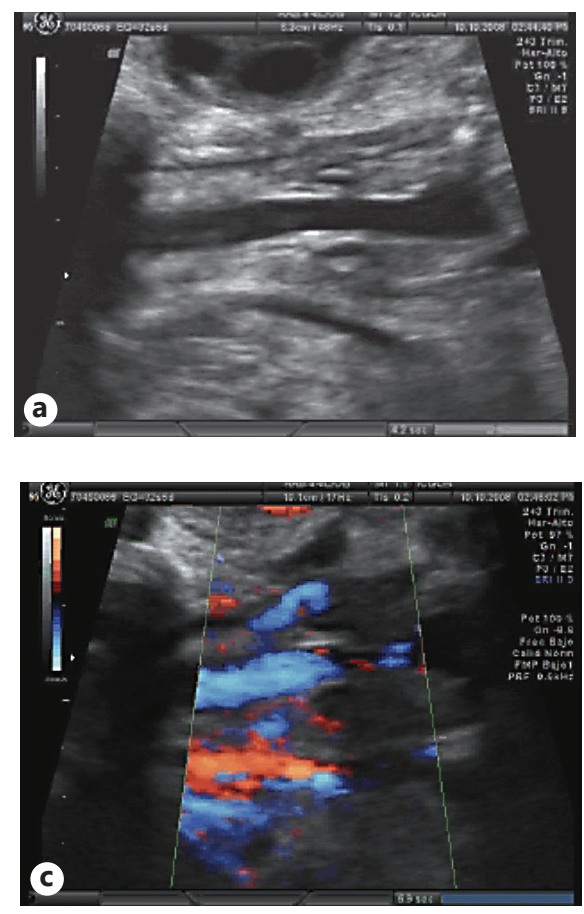

Fig. 1. Coronal view of the fetal neck at the level of the trachea, showing normal appearance of the larynx and vocal cords with normal movements of opening (a) and closure (b). Documentation of passage (c) in and out of fluid (d) by color Doppler.

Fig. 2. Abnormal aspect of the fetal larynx suggestive of laryngeal atresia/dysplasia. The normal appearance of the larynx and vocal cords is not observed. The cords appear echogenic and conglutinated (a), and no opening-closure movements can be documented (b).

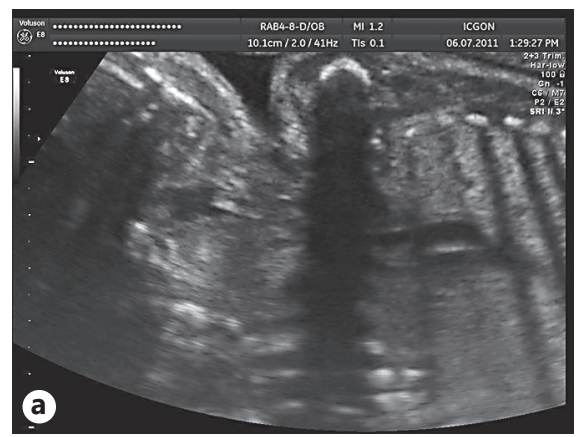

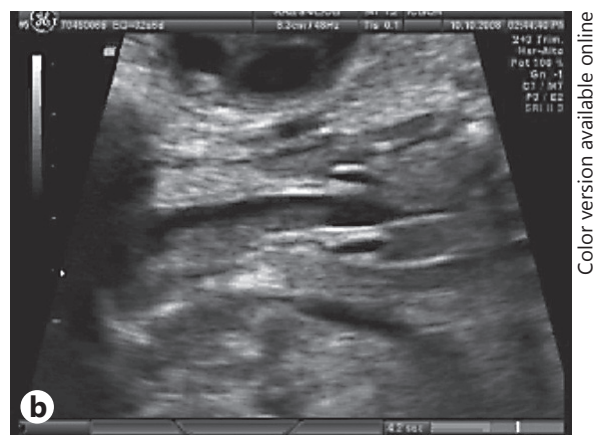
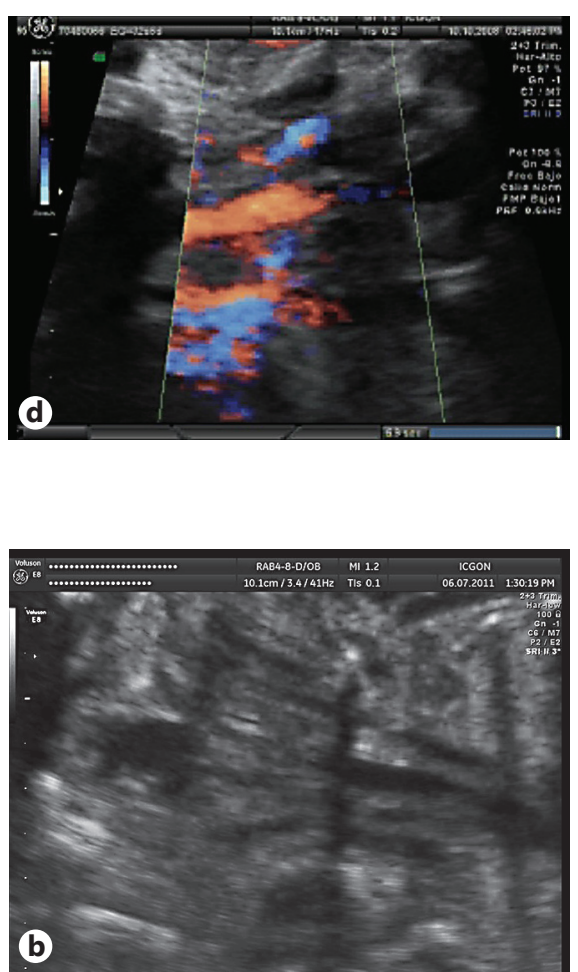

chea and the lower end of the pharynx in the same view, it was impossible to visualize the normal aspect of the lar$y n x$. The area where the larynx is normally visualized was occupied by ill-defined nonstructured tissue. Several important anatomical landmarks were missing, including obviously the normal appearance of the vocal cords bulging in the hypoechogenic pharynx. In addition, the characteristic tapering of the uppermost end of the trachea as it merges with the larynx was missing and instead the trachea was ended abruptly. In addition, it was obviously impossible to observe any opening-closure movements at this level (fig. 2) and no tracheal fluid movement could be demonstrated with Doppler whatsoever. Termination of pregnancy was performed in 6 cases on parental request because of the poor prognosis of the condition. Two cases had associated anomalies: double inlet single ventricle with transposition of the great arteries, Fraser syndrome (unilateral renal agenesis with contralateral hydronephrosis and microphthalmia). The prenatal diagnosis of laryngeal atresia was confirmed in all these cases by necropsy (fig. 3).

Case 7 was the first pregnancy of a 38-year-old mother, with no history of medications during the first trimester, whose family history did not reveal congenital anomalies, hereditary diseases or consanguinity. A transvaginal first-trimester scan was normal and combined screening for Down's syndrome showed low risk. At 21 weeks' gestation, the diagnosis of CHAOS with hydrops 
Fig. 3. Macroscopic transverse section of a dysplasic laryngeal atresia in a 20-week fetus undergoing termination of pregnancy (a) at the level of vocal cords, which in this case appear thickened and dysplastic, and (b) just below, there is no visible laryngeal lumen, but instead there is a fibrous tissue with conglutinated vocal cords.

Fig. 4. Diagnosis of CHAOS in the treated case at 21 weeks' gestation. Massive bilateral lung enlargement and hyperechogenicity, with inverted diaphragms (a, b), severe ascites (c), and dilation of the airways (d).
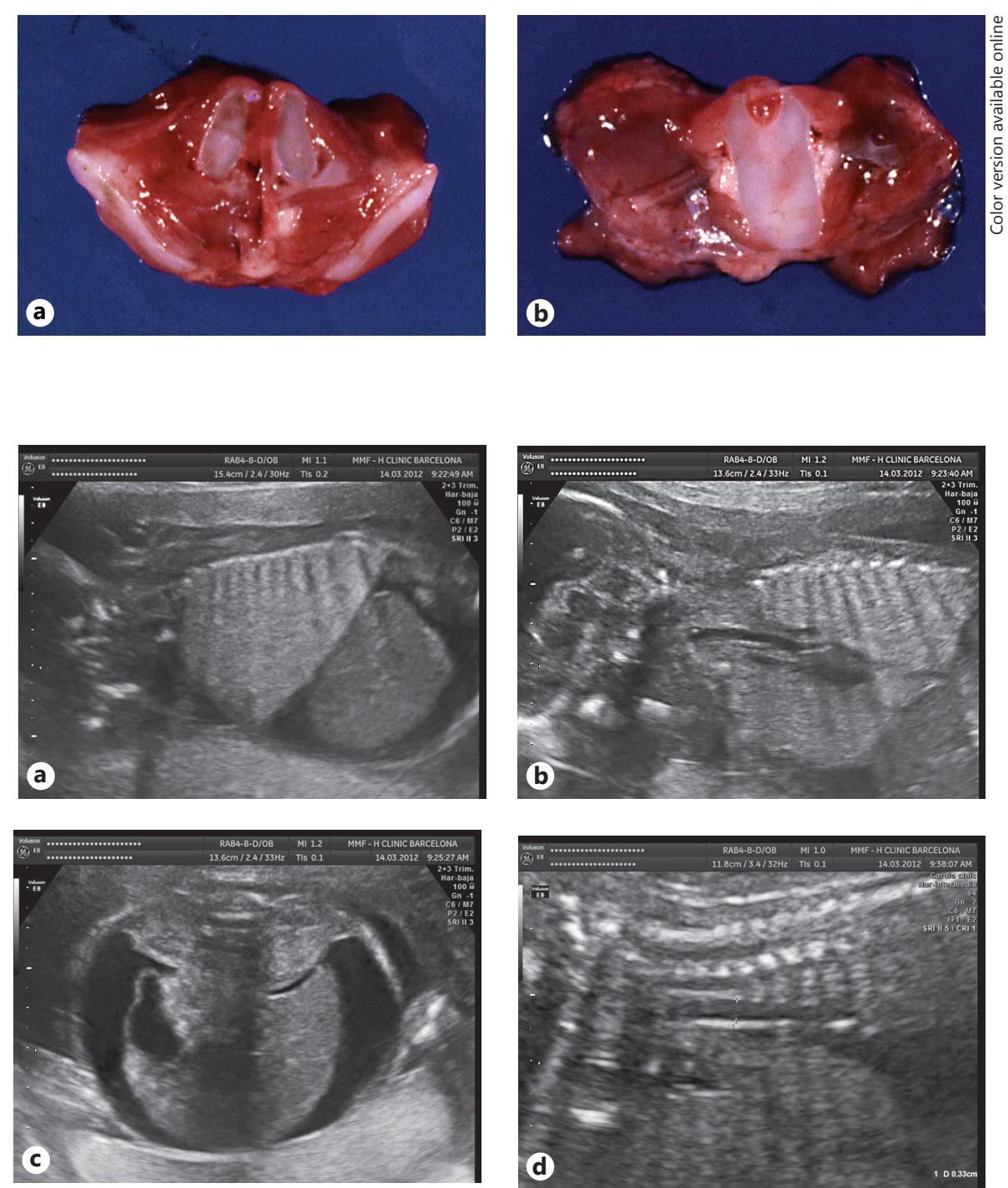

fetalis (generalized skin edema of $15 \mathrm{~mm}$ and severe ascites) was detected (fig. 4). In spite of maternal obesity, a fair visualization on ultrasound scan, the appearance of the trachea was normal, with a diameter of $3 \mathrm{~mm}$. In addition, there was a normal appearance of the vocal cords, which showed opening-closure movements periodically (fig. 5). However, no passage of fluid through the trachea by color Doppler could be demonstrated. A full anatomical scan by ultrasound and magnetic resonance imaging ruled out associated other fetal anomalies. Fetal growth was normal and karyotype was 46,XX. After extensive counseling and local ethics committee approval, parents accepted and gave informed consent for a fetoscopic surgery approach.
Fetal surgery was performed at 21.2 weeks by means of a similar procedure to FETO for $\mathrm{CDH}$ [9]. Under maternal epidural and fetal intramuscular analgesia and immobilization as previously described, a 3-mm trocar was percutaneously introduced into the amniotic cavity and directed towards the mouth of the fetus. A normal pharynx and epiglottis were confirmed. The fetoscope was then advanced towards the trachea, and normal vocal cords could be observed. Just below the vocal cords, a bulging tissue occluding the tracheal lumen could be observed (fig. 6). At that time, ultrasound visualization confirmed that directly beneath the tip of the scope, which was facing the bulging tissue, there was the dilated trachea (fig. 7). The membranous tissue was then gently touched with a diode laser fiber at a power settings of $10 \mathrm{~W}$, and it im-

Martínez/Castañón/Gómez/Prat/ Eixarch/Bennasar/Puerto/Gratacós 
Fig. 5. Same fetus as in figure 4. Normal appearance of the vocal cords showed opening (a) and closure (b) movements periodically.

Fig. 6. Fetoscopic view of the vocal cords $(\mathrm{V})$, the beginning of the tracheal lumen (T) and immediately behind a bulging membrane (B). E = External sheath of the trocar.

Fig. 7. Intraoperative ultrasound visualization of the dilated trachea directly beneath the tip of the fetoscope.

Fig. 8. Fetoscopic view of the lumen of the vocal cords after puncture of the obstructing membrane. The tracheal lumen is open. The remains of the web-like membrane can be seen attached to the posterior aspect of the trachea (left upper part of the tracheal lumen in the picture). A small amount of blood and epithelial injury is seen in the anterior aspect (right lower part in the picture).

Fig. 9. Ultrasound evaluation $24 \mathrm{~h}$ after surgery. Remarkable decrease in the echogenicity of the lung which had a virtually normal appearance with horizontalized diaphragms.
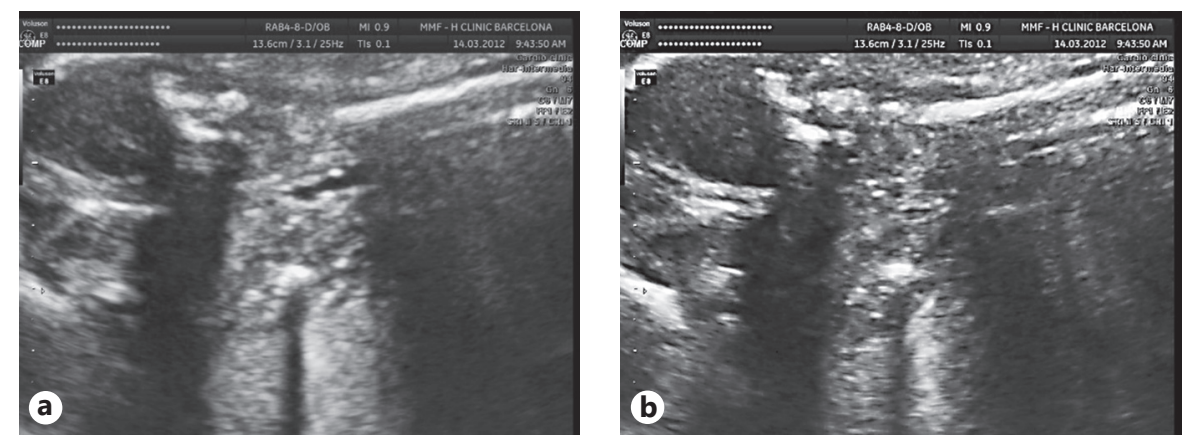

6
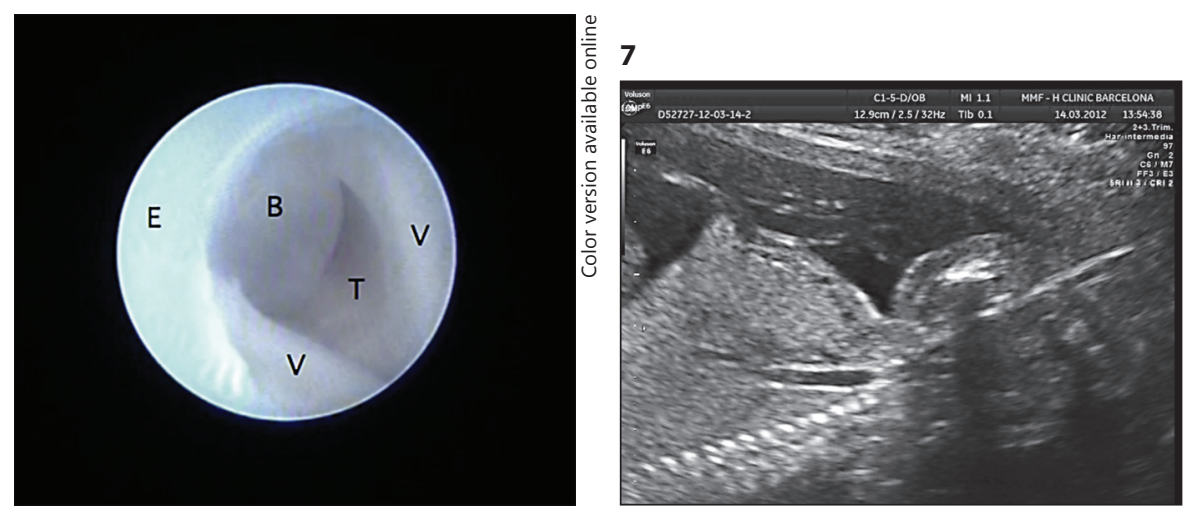

8
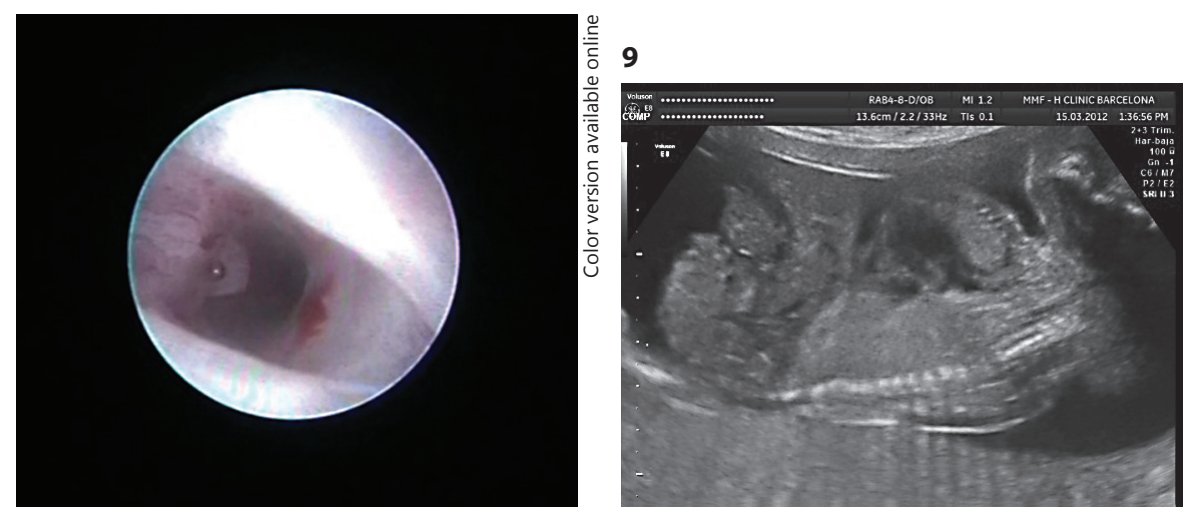

mediately blew up allowing excretion of a large amount of dense mucous secretion (fig. 6). Of note, this secretion was remarkably similar in appearance to that observed after withdrawal of a tracheal balloon following FETO for $\mathrm{CDH}$. The remains of the web-like membrane could be observed attached to the tracheal wall about $1 \mathrm{~cm}$ below the vocal cords (fig. 8). Endoscopic inspection confirmed an apparently normal trachea, carina and bronchi, and the fetoscopic procedure was finalized. The whole proce- dure lasted 22 min skin-to-skin with no changes in fetal heart rate at any time.

Over the minutes following the surgery, a rapid and remarkable decrease in the size and echogenicity of the lungs was observed. One day after surgery, the lungs appeared virtually normal, the tracheobronchial tree was no longer dilated, and both diaphragms had horizontalized (fig. 9). The patient was discharged home $48 \mathrm{~h}$ after surgery. Five days after surgery, though ascites persisted, 
10

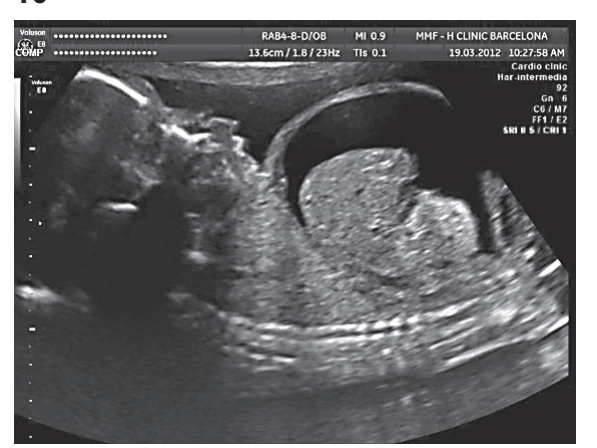

11

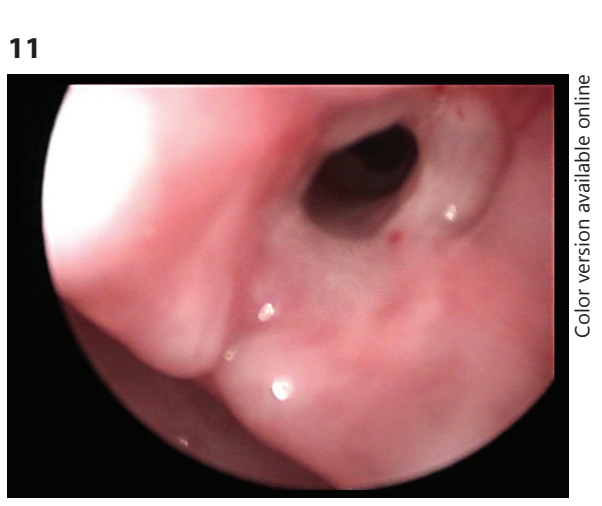

Fig. 10. Five days after surgery, lungs and diaphragms looked normal, skin edema had almost disappeared and ascites persisted.

Fig. 11. Bronchoscopy at 3 months of life identified a normal larynx, vocal cords and trachea, with no remnants of the subglottic membrane. skin edema had almost disappeared and diaphragms looked normal (fig. 10). Over the next weeks after the procedure, a significant improvement in hydropic findings was observed. Skin edema disappeared 2 weeks and ascites 8 weeks after the procedure. The course of pregnancy was otherwise uneventfully.

A male infant with a birth weight of 2,850 $\mathrm{g}$ was born at 37 weeks' gestation after spontaneous vaginal delivery, with an Apgar score of 10 at $5 \mathrm{~min}$. The newborn did not require any type of ventilatory support. Thoracic X-ray and computed tomography scan showed normal lungs. The infant was discharged at 5 days. Bronchoscopy at 3 months of life identified a normal larynx, normally moving vocal cords and trachea (fig. 11). No remnants of the subglottic membrane could be observed. At the time of this writing the infant was 9 months old, healthy, and neurocognitive development was normal.

\section{Discussion}

This series demonstrates that systematic ultrasound visualization of the vocal cords can be reliably performed in fetuses diagnosed with CHAOS. The diagnosis of CHAOS usually bears dismal consequences because of the anomaly of the larynx by itself, or because of the common presence of associated anomalies or hydrops fetalis $[1,2]$. However, a good outlook has been occasionally described in some cases, thus the challenge for fetal medicine specialists has become to identify prenatal factors predictive not only of infant survival [4] but also of a future correct functioning of the larynx with a normal quality of life. We here provide evidence that ultrasound might be of great help in selecting the small subset of fetuses with CHAOS due to isolated upper tracheal obstruction but otherwise normal larynx, which may benefit from fetoscopic surgery. Very recently, in line with our argument, an almost $90 \%$ successful evaluation of the fetal pharynx and larynx at 20-24 weeks' gestation by combined use of $2 \mathrm{D}$ and $3 \mathrm{D}$ ultrasound based on 5 spatial planes has been reported [13]. The authors suggest a potential role for their methodology in the evaluation and management of certain pathologies, such as CHAOS [13].

Only six attempts of fetal therapy for CHAOS have been reported [5-8, 14]. In the first case, Ward et al. [5] attempted an open surgery fetal tracheostomy at 18 weeks' gestation, but there was a fetal death a few hours later. In the second case, the uterus was exteriorized through a transverse laparotomy and three 5-mm trocars were used. Tracheal dissection was attempted but during the procedure, the fetus developed severe bradycardia and eventually was delivered by EXIT procedure. After 6 months, the baby was discharged on assisted ventilation with permanent tracheostomy. At 4 years of age, the child suffered severe sequelae, including no speech, a permanent gastrostomy, assisted ventilation at night and mild neurodevelopmental delay [6]. In the third case, Kohl et al. [7] performed a fetoscopic perforation of a tracheal atresia in a hydropic fetus. The baby was diagnosed as having Fraser syndrome after birth. Surgery consisted of a three 5 -mm port procedure which lasted 215 min under general anesthesia. The patient experienced premature delivery at 28 weeks' gestation. Pulmonary function was correct and the baby was discharged from hospital at 6 months of age, with severe sequelae [7, 15]. Later, the same group performed a percutaneous fetoscopic tracheal decompression via a unique $5-\mathrm{mm}$ port under general anesthesia at 22 weeks' gestation. The procedure lasted $165 \mathrm{~min}$. There was no complete normalization of the aspect of the lungs or the diaphragms, and ascites and oligoanhydramnios persisted until delivery. The fetus was delivered by EXIT tracheotomy at 31 weeks' gestation. Unfortunately, diaphragmatic function remained im- 
paired so that tracheotomy and ventilatory support were continued beyond discharge from hospital at 4 months of age. The infant died at home 2 weeks later [8]. Finally, Saadai et al. [14] reported 2 cases which underwent fetal bronchoscopy with an attempted wire tracheoplasty, i.e. a stiff guidewire that punctures the atretic segment of the trachea and is then removed. Thereafter, both were delivered via EXIT. One of them showed associated major anomalies postnatally that had not been noticed during pregnancy, and at 13 months of age was tracheostomyand ventilator-dependent, and feeding by gastrostomy. The other case was presented at postnatal tracheoscopy with a tracheoesophageal fistula, and after several postnatal interventions the infant is free of tracheostomy, feeds by mouth and is able to phonate at 3 years of age. However, the authors acknowledge that they were not sure of having decompressed the airway during the fetal intervention [14].

Unlike these previous reports, in our case we could clearly document a membrane-like structure directly below the vocal chords. In these cases, fetoscopic deobstruction offers a curative approach. The prenatal intervention can result in a huge improvement in terms of postnatal evolution. Indeed, fetuses with CHAOS and hydrops may survive for a long time in utero, so that they often reach viable gestational ages to allow delivery assisted by EXIT. However, as confirmed by previous reports, in spite of this approach, perinatal mortality remains high [1-4]. In the surviving cases, the quality of lung tissue is poor and rarely allows normal respiratory function, possibly due to dysplastic features resulting from long-term exposure to high airway pressure. The long-term evolution of these children is also associated with exceedingly high morbidity and poor quality of life $[4,7,8]$. According to the small available evidence, if CHAOS is caused by isolated subglottic membrane, fetoscopy can be a short single-port curative procedure, which permits normalization of lung development. Our case shows for the first time an infant with normal postnatal respiratory function who remains completely healthy at 9 months of age.

The use of fetoscopy in CHAOS adds evidence to support the use of this form of fetal therapy in selected forms of upper airway obstruction. We recently described successful fetoscopic airway decompression in a fetus affected of bilobar bronchial atresia presenting in the form of a massive right lung tumor causing severe thoracic compression with mediastinal shift, pulmonary hypoplasia and fetal hydrops [16]. Extended clinical experience with FETO for CDH [12] has allowed gaining confidence in fetal airway endoscopy as a safe and reproducible procedure, which can now be successfully extended to rare indications, as illustrated by these cases.

In summary, in this study we provide evidence that laryngeal anatomy can be reliably observed in cases of CHAOS, facilitating parents' counseling and planning of therapy in very selected cases. As mentioned in previous reports $[12,16]$, we must stress again that these exceptional and complex techniques must be only offered by highly experienced multidisciplinary fetal surgery teams, with high-level postnatal neonatal and pediatric surgery care. If these requirements are respected, the fetoscopic approach may represent a life-saving opportunity for a small subset of fetuses with CHAOS worldwide every year.

\section{References}

1 Hedrick MH, Ferro MM, Filly RA, Flake AW, Harrison MR, Adzick NS: Congenital high airway obstruction syndrome (CHAOS): a potential for perinatal intervention. J Pediatr Surg 1994;29:271-274.

$\checkmark 2$ Cavoretto P, Molina F, Poggi S, Davenport M, Nicolaides KH: Prenatal diagnosis and outcome of echogenic fetal lung lesions. Ultrasound Obstet Gynecol 2008;32:769-783.

$>3$ Berg C, Geipel A, Germer U, et al: Prenatal detection of Fraser syndrome without cryptophthalmos: case report and review of the literature. Ultrasound Obstet Gynecol 2001;18: 76-80.

$\checkmark 4$ Roybal JL, Liechty KW, Hedrick HL, Bebbington MW, Johnson MP, Coleman BG, Adzick NS, Flake W: Predicting the severity of congenital high airway obstruction syndrome. J Pediatr Surg 2010;45:1633-1639.
5 Ward VMM, Langford K, Morrison G: Prenatal diagnosis of airway compromise: EXIT (ex utero intra-partum treatment) and foetal airway surgery. Int J Pediatr Otorhinolaryngol 2000;53:137-141.

6 Paek BW, Callen PW, Kitterman J, Feldstein VA, Farrell J, Harrison MR, Albanese CT: Successful fetal intervention for congenital high airway obstruction syndrome. Fetal Diagn Ther 2002;17:272-276.

$>7$ Kohl T, Hering R, Bauriedel G, Van de Vondel P, Heep A, Keiner S, Muller A, Franz A, Bartmann P, Gembruch U: Fetoscopic and ultrasound-guided decompression of the fetal trachea in a human fetus with Fraser syndrome and congenital high airway obstruction syndrome (CHAOS) from laryngeal atresia. Ultrasound Obstet Gynecol 2006;27:8488.
>8 Kohl T, Van de Vondel P, Stressig R, Wartenberg HC, Heep A, Keiner S, Muller A, Franz A, Fröhlich S, Willinek W, Gembruch U: Percutaneous fetoscopic laser decompression of congenital high airway obstruction syndrome (CHAOS) from laryngeal atresia via a single trocar. Current technical constraints and potential solutions for future interventions. Fetal Diagn Ther 2009;25:67-71.

$>9$ Vidaeff AC, Smuk P, Mastrobattista JM, Rowe TF, Ghelber O: More or less CHAOS: case report and literature review suggesting the existence of a distinct subtype of congenital high airway obstruction syndrome. Ultrasound Obstet Gynecol 2007;30:114-117. 
>10 Lim FY, Crombleholme TM, Hedrick HL, Flake AW, Johnson MP, Howell LJ, Adzick NS: Congenital high airway obstruction syndrome: natural history and management. J Pediatr Surg 2003;38:940-945.

11 Mong A, Johnson AM, Kramer SS, et al: Congenital high airway obstruction syndrome: MR/US findings, effect on management, and outcome. Pediatr Radiol 2008;38:1171-1179.
2 Deprest JA, Nicolaides K, Gratacos E: Fetal surgery for congenital diaphragmatic hernia is back from never gone. Fetal Diagn Ther. 2011;29:6-17.

13 Liberty G, Boldes R, Shen O, Shaul C, Cohen SM, Yagel S: The fetal larynx and pharynx: structure and development described by $2 \mathrm{D}$ and 3D ultrasound examination. Ultrasound Obstet Gynecol (E-pub ahead of print).

14 Saadai P, Jelin EB, Nijagal A, Schecter SC, Hirose S, MacKenzie TC, Rand L, Goldstein R, Farrell J, Harrison M, Lee H: Long-term outcomes after fetal therapy for congenital high airway obstructive syndrome. J Pediatr Surg 2012;47:1095-1100.
15 Ville Y: Editor's note. Ultrasound Obstet Gynecol 2006;27:88.

16 Martínez JM, Prat J, Gómez O, Crispi F, Bennasar M, Puerto B, Castañón M, Gratacós E: Decompression through tracheobronchial endoscopy of bronchial atresia presenting as massive pulmonary tumor: a new indication for fetoscopic surgery. Fetal Diagn Ther 2013; 33:69-74. 